OPEN ACCESS

Edited by:

Sunjoo Kang,

Yonsei University, South Korea

Reviewed by:

Bussma Bugis,

Saudi Electronic University,

Saudi Arabia

Nilesh Chandrakant Gawde,

Tata Institute of Social Sciences, India

*Correspondence:

Noor Al Khathlan

Naalkhathlan@iau.edu.sa

tThese authors have contributed equally to this work

Specialty section:

This article was submitted to

Public Health Education and

Promotion,

a section of the journal

Frontiers in Public Health

Received: 27 May 2021

Accepted: 19 July 2021

Published: 18 August 2021

Citation:

Al Khathlan N and Padhi BK (2021) Adherence to COVID-19 Appropriate

Behavior Among Respiratory

Therapists: A Cross-Sectional Study in the Kingdom of Saudi Arabia.

Front. Public Health 9:715982. doi: 10.3389/fpubh.2021.715982

\section{Adherence to COVID-19 Appropriate Behavior Among Respiratory Therapists: A Cross-Sectional Study in the Kingdom of Saudi Arabia}

\author{
Noor Al Khathlan ${ }^{1 * t}$ and Bijaya Kumar Padhi ${ }^{2+}$ \\ ${ }^{1}$ Department of Respiratory Care, College of Applied Medical Sciences, Imam Abdulrahman Bin Faisal University, Dammam, \\ Saudi Arabia, ${ }^{2}$ Department of Community Medicine \& School of Public Health, Post Graduate Institute of Medical Education \\ and Research, Chandigarh, India
}

Background: Adherence to novel coronavirus disease 2019 (COVID-19) appropriate behavior plays a crucial element in the management of the infections of COVID-19. Despite the importance of transmission-reducing behaviors among healthcare professionals, there is a lack of literature in this area of research explicitly relating to respiratory therapists (RTs). Therefore, it is essential to assess the adherence level to COVID-19 transmission-reducing behaviors among the RTs in Saudi Arabia.

Methods: A web-based online survey was conducted using questions based on the risk assessment guidelines of WHO. A random representative sample of RTs $(N=215)$ residing in Saudi Arabia was recruited for the study. Descriptive and inferential statistics were computed using STATA software. Logistic regression analysis was used to identify key factors that are associated with adherence to COVID-19 appropriate behavior among the study participants.

Results: Of the 215 participants, 59.5\% were aged between 26 and 35 years, and $40.9 \%$ were women. Most (85.5\%) participants had a bachelor's degree while $12.0 \%$ had a master's degree. About $56.2 \%$ of RTs provided direct care to a confirmed patient of COVID-19 during the study periods. The study showed $80.9 \%$ of RTs in Saudi Arabia adhered to personal protective equipment (PPE) at the workplace and $65.0 \%$ at home. Moreover, the findings of the study indicated that senior RTs (with $>5$ years of experience) demonstrated a higher adherence level to the guidelines than RTs with $<5$ years of experience. High-risk perception [aOR:2.32; 95\% Cl: 1.09-3.27], and work history of $<5$ years [aOR:2.00; 95\% Cl: 1.14-3.15], were found to be the strongest predictors in explaining the adherence to appropriate behavior among the RTs at the workplace. Whereas the high-risk perception [aOR:2.32; 95\% Cl: 1.09-3.27] and being married [aOR:1.85; 95\% Cl: 1.08-3.82] were found to be the strongest predictors at home.

Conclusion: Adherence ("Always" or "Most times") to COVID-19 appropriate behavior was found to be high at hospital settings among the study participants. However, the same practice was found to be inconsistence in non-healthcare settings among the 
RTs. Considering the paramount role of COVID-19 appropriate behavior in reducing the transmissions the policy focus, therefore, needs to be on creating a well-spread behavior change communication that is curtailing the adoption of appropriate behavior in the non-healthcare settings.

Keywords: respiratory care, respiratory therapist, COVID-19 protective behavior, transmission-reducing behaviors, Saudi Arabia, COVID-19 appropriate behavior

\section{BACKGROUND}

The novel coronavirus disease 2019 (COVID-19) has left the world reeling, the effects on the public health systems across the world has been devastating. It has posed significant challenges for the healthcare workers, and their safety thus must be ensured. Respiratory care (RC) is an important medical specialty which deals with the diagnosis and treatment of patients with acute and chronic respiratory diseases. Respiratory therapists (RTs) work alongside doctors in the various areas such as intensive care units (ICUs), emergency departments, out-patient departments, and home care settings. Involvement of RT in caring patients with COVID-19 is found to be extremely vital at this juncture $(1,2)$. Their expertise in assessing blood oxygen levels, assisting with intubations and bronchoscopies, and specializing in management of ventilator has been extremely valuable, and sometimes exposed them to the sickest patients and to dangerous aerosolizing procedures. In view of the importance of the specialty of RC and the effective role of an RT, public and private universities in the Kingdom have begun to offer RC programs since the 1988 (3). Though the RC profession itself has been recognized in Saudi Arabia over 40 years, but still not fully appreciated in the Saudi hospitals (3). Currently, around 10 universities offer $\mathrm{RC}$ programs. It has been reported that out of 411 hospitals in the country, only 88 hospitals offer services by the trained RTs, which represent $21.4 \%$ of total number of working hospitals in the country (4). As of December 2020, the total number of active RTs in Saudi Arabia is 1,477, one RT for every 17,629 population of Saudi (4). Half of them, work in the central part of the country and one quarter are Saudi nationals (4). Furthermore, majority of the RT workforce in the Kingdom, work in critical care settings which represents about $44.5 \%$ (4).

Numerous studies have reported inadequate training on infection prevention and control, and shortages of personal protective equipment (PPE) resulting in high rates of COVID19 among healthcare workers $(5,6)$. In China, a study reported inadequate $\mathrm{PPE}$ as a reason for infection among healthcare workers (7).A systematic reviews and meta-analysis estimated that $21.7 \%$ of the healthcare workers experienced depression, $21.5 \%$ PTSD, and $22.1 \%$ anxiety during the pandemic (8). The Middle East reported the highest pooled rates of depression (34.6\%) and anxiety (28.9\%) (8).

As RTs continue to run the ventilators that are keeping many patients with COVID-19 alive, majority of procedures that therapists perform occur at the head of the bed of the patients, putting therapists at a high risk for infection. Adherence to COVID-19 appropriate behavior plays a crucial element in the management of the infections of COVID-19. Despite the importance of adherence to the guidelines of COVID-19, there is a lack of literature in this field of research specifically relating to RTs. However, in the last year, RTs have been playing a major role in treating patients hospitalized with COVID-19. RTs have often been overlooked during the COVID-19 epidemic, even though they have played a crucial role. Therefore, it is essential to assess the risk perception and adherence level to the guidelines of COVID-19 among RTs in Saudi Arabia. The study was planned to assess the risk perception and adherence level of RTs to the guidelines of COVID-19 in Saudi Arabia.

\section{METHODS}

\section{Ethics Consideration}

Ethical approval was obtained from Deanship of Scientific Research (DSR) at Imam Abdulrahman Bin Faisal University (IAU), Dammam, Saudi Arabia. Informed consent was provided to all the survey participants prior to their enrolment. Participants were allowed to terminate the survey at any time they desire. The survey data analysis and reporting ensured compliance with ethical requirements of Imam Abdulrahman Bin Faisal University (IAU), Dammam, Saudi Arabia.

\section{Study Design and Sample}

The study was a cross-sectional, hospital-based survey conducted via simple random sampling. The study was conducted between March and June 2020 (the first phase of pandemic). RTs ( $N$ $=215$ ) were randomly sampled from the list of RTs (as of December 2019) in the country and were asked to participate in this study. We collected the email address, and phone numbers of RTs working in various departments of RC across the country. The survey link was shared to them those who consented to participate in the survey. The target sample size of the participants was determined using the formula, $N=\mathrm{Z} \alpha 2 \mathrm{P}(1-\mathrm{P})$ $/ \mathrm{d} 2$, in which $\alpha=0.05$ and $\mathrm{Z} \alpha=1.96$, and the estimated acceptable margin of error for proportion $d$ was $5 \%$. The proportion of RTs with COVID-19 appropriate behaviors was estimated at $80 \%$, based on the available literature on infection control behaviors among healthcare professionals (9-11). The sample size was calculated using OpenEpi software (v3.01).

\section{Development of Study Questionnaires}

A draft version of the survey was developed by the research team based on questionnaires of the WHO risk assessment guideline (12), and constructs of health predictive models (13-16). The draft questionnaires were validated and tested measures where 
possible, and then shared with the expert for their opinion. The draft survey was modified based on the feedback received to ensure question wording was clear and easily understood. A bilingual survey (Arabic and English) was shared to study participants to record their response.

TABLE 1 | Adherence to COVID-19 appropriate behavior at workplace $(N=215)$.

Variables
Wear personal protective equipment (PP
Always as recommended
Most of the times
Occasionally
Single gloves
Always as recommended
Most of the times
Occasionally
Rarely
Medical mask
Always as recommended
Most of the times
Occasionally
Rarely
Face shield or goggles/protective glasses
Always as recommended
Most of the times
Occasionally
Rarely
Disposable Gown
Always as recommended
Most of the times
Occasionally
Rarely

$n(\%)$

Remove and replace PPE according to protocol ${ }^{\star}$

Always as recommended

Most of the times

Occasionally

Rarely

Before and after touching the COVID-19 patient?

Always as recommended

Most of the times

Rarely

Before and after any clean or aseptic procedure

Always as recommended

Most of the times

Occasionally

Rarely

After exposure to body fluid?

Always as recommended

Most of the times

Occasionally

Rarely

After touching the COVID-19 patient's surroundings

Always as recommended

Most of the times

Occasionally

Rarely

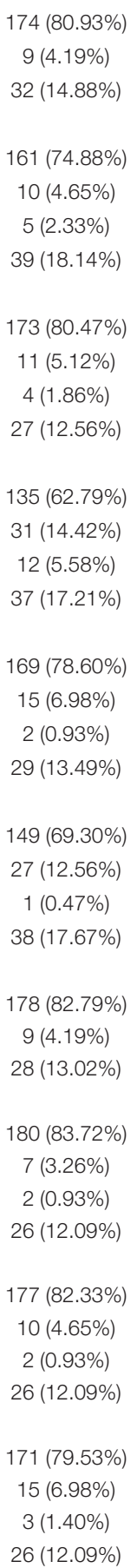

TABLE 2 | Adherence to COVID-19 appropriate behavior other than healthcare setting $(N=215)$.

Variables $n(\%)$

\section{Disinfect home}

Always as recommended

$118(54.88 \%)$

Most of the times

$58(26.98 \%)$

Occasionally

$31(14.42 \%)$

Rarely

$8(3.72 \%)$

Wash hands frequently with soap or hand sanitizer

Always as recommended

$169(78.60 \%)$

Most of the times

$40(18.60 \%)$

Occasionally

$6(2.79 \%)$

Cover mouth and nose when coughing or sneezing

Always as recommended

$177(82.33 \%)$

Most of the times

$32(14.88 \%)$

Occasionally

$5(2.33 \%)$

Rarely

$1(0.47 \%)$

Wear mask

Always as recommended

$102(47.44 \%)$

Most of the times

$54(25.12 \%)$

Occasionally

$37(17.21 \%)$

Rarely

$22(10.23 \%)$

Avoided public transportation

Always as recommended

$172(80.00 \%)$

Most of the times

$32(14.88 \%)$

$7(3.26 \%)$

$4(1.86 \%)$

Rarely

$\begin{array}{lc}\text { Always as recommended } & 149(69.30 \%) \\ \text { Most of the times } & 51(23.72 \%)\end{array}$

Avoided social activities

Most of the times

$8(3.72 \%)$

$\begin{array}{ll}\text { Rarely } & 7(3.26 \%)\end{array}$

Avoided going to hospital/clinic

Always as recommended $\quad 114(53.02 \%)$

Most of the times $\quad 40(18.60 \%)$

Occasionally $18(8.37 \%)$

Rarely $43(20.00 \%)$

Avoided contact with people who have recently had a travel history

Always as recommended $156(72.56 \%)$

Most of the times $\quad 47(21.86 \%)$

Occasionally $\quad 8(3.72 \%)$

Rarely $4(1.86 \%)$

Avoided going to crowded places

Always as recommended $\quad 143(66.51 \%)$

Most of the times $\quad 47(21.86 \%)$

Occasionally 12 (5.58\%)

Rarely $13(6.05 \%)$

Avoided contact with people who had fever or symptoms of respiratory illness

$\begin{array}{lc}\text { Always as recommended } & 175(81.40 \%) \\ \text { Most of the times } & 24(11.16 \%) \\ \text { Occasionally } & 10(4.65 \%) \\ \text { Rarely } & 6(2.79 \%) \\ \text { Avoided Shaking Hands } & \\ \text { Always as recommended } & 180(83.72 \%) \\ \text { Most of the times } & 16(7.44 \%) \\ \text { Occasionally } & 14(6.51 \%) \\ \text { Rarely } & 5(2.33 \%)\end{array}$




\section{Outcomes and Covariates}

The study focused on adherence to COVID-19 appropriate behaviors that reduce transmission of COVID-19. Adherence to each behavior was assessed using a 5-point response scale (always, most times, sometimes, rarely, never) in two occasions (hospital duty and non-hospital settings). Respondents were asked to record the extent to which they had adhered to each behavior over the previous week. The covariates, sociodemographic characteristics such as age, gender, nationality, hospital type (private vs. government), geographical region, and education status were assessed. Risk perception was assessed using a threepoint response scale "Very Concerned" (High-risk perception); "Somewhat Concerned" (Moderate-risk perception); and "Not at all Concerned" (Low-risk perception) to the question "How concerned are you that you or someone in your family will be infected with COVID-19 virus?"

\section{Statistical Analysis}

The anonymized data were further categorized, sex (male or female), age $(18-25,26-30,31-40$, or $>40$ years), marital status (married or single), educational level ( $\leq$ undergraduate or $\geq$ postgraduate), technical title (junior, intermediate, or senior), geographic location (East, West, North, South, and Central province), nationality (Saudi or foreign), and type of hospital (secondary or tertiary). RTs were asked to record whether they were directly engaged in the clinical activities or providing care to patients with COVID-19. A composite variable "adherence to COVID-19 appropriate behaviors" was constructed by dichotomizing all key variables mentioned in Tables 1, 2. From the Likert response questions, the top two responses (e.g. "always" and "most times") received a "1" and the three other responses (e.g., "sometimes," "rarely," or "never") received a " 0 ".

To determine potential predictive factors for adherence to COVID-19 appropriate behavior among participants, multivariable logistic regression analysis was performed. Model estimates were presented as ORs and 95\% CIs, after adjustment for confounders, including sex, age, marital status, educational level, technical title, place of residence, and type of hospital. Data analysis was performed using STATA statistical software version 15.0 (STATA Corp.). The significance level was set at $\alpha=0.05$, and all tests were two-tailed.

\section{RESULTS}

A total of 215 RC professionals participated in this study. Of the 215 participants, $59.5 \%$ were aged $26-35$ years, $40.9 \%$ were female and most of them had completed bachelor's degree. About $20 \%$ were of non-Saudi nationality, and half of them were having work history of more than 5 years. Approximately $56.2 \%$ provided direct care to a confirmed COVID-19 patient, and about $47.9 \%$ reported in engaging aerosol-generating procedures (AGP). About half of them were from government hospital under Ministry of Health, and $54.8 \%$ belonged to the eastern provinces of the country (Table 3 ).

Table 1 presents participants adherence to COVID-19 appropriate behavior at workplace. Most (80.9\%) of the
TABLE 3 | Socio-demographic characteristics, and risk perception to COVID-19 of the study participants $(N=215)$.

\begin{tabular}{|c|c|}
\hline Variables & $n(\%)$ \\
\hline \multicolumn{2}{|l|}{ Age (Years) } \\
\hline $18-25$ & $34(15.81 \%)$ \\
\hline $26-35$ & $128(59.53 \%)$ \\
\hline Above 35 & $53(24.65 \%)$ \\
\hline \multicolumn{2}{|l|}{ Gender } \\
\hline Male & $127(59.07 \%)$ \\
\hline Female & 88 (40.93\%) \\
\hline \multicolumn{2}{|l|}{ Highest education } \\
\hline $\mathrm{PhD}$ & $5(2.33 \%)$ \\
\hline Masters & $26(12.09 \%)$ \\
\hline Bachelor & $184(85.58 \%)$ \\
\hline \multicolumn{2}{|l|}{ Nationality } \\
\hline Saudi & $173(80.47 \%)$ \\
\hline Non-Saudi & $42(19.53 \%)$ \\
\hline \multicolumn{2}{|l|}{ Years of working experience as RT } \\
\hline$>10$ Years & $65(30.23 \%)$ \\
\hline $6-10$ Years & $40(18.60 \%)$ \\
\hline $1-5$ Years & $86(40.00 \%)$ \\
\hline$<1$ Year & $24(11.16 \%)$ \\
\hline \multicolumn{2}{|l|}{ Type of hospital } \\
\hline $\mathrm{MOH}-$ Governmental hospital & $105(48.84 \%)$ \\
\hline Non-MOH Governmental hospital & $83(38.60 \%)$ \\
\hline Private hospital & $27(12.56 \%)$ \\
\hline \multicolumn{2}{|l|}{ Hospital geographical location } \\
\hline Eastern Provinces & $118(54.88 \%)$ \\
\hline Western Provinces & $38(17.67 \%)$ \\
\hline Southern Provinces & $8(3.72 \%)$ \\
\hline Central Provinces & $51(23.72 \%)$ \\
\hline \multicolumn{2}{|c|}{ Direct care to a confirmed COVID-19 patient } \\
\hline No & $94(43.72 \%)$ \\
\hline Yes & $121(56.28 \%)$ \\
\hline \multicolumn{2}{|c|}{ Present when any aerosol generating procedures (AGP) } \\
\hline No & $112(52.09 \%)$ \\
\hline Yes & $103(47.91 \%)$ \\
\hline \multicolumn{2}{|l|}{ Risk Perception } \\
\hline Low & $41(19.06 \%)$ \\
\hline Moderate & $60(27.90 \%)$ \\
\hline High & 114 (53.02\%) \\
\hline
\end{tabular}

study participants reported that they always wear PPE as recommended. Of the 215 respondents, $74.8 \%$ always used single gloves, $80.4 \%$ always used medical mask, $62.7 \%$ always used face shield, $62.7 \%$ always used disposable gown. About two-third of the study participants reported that they removed and replaced PPE according to protocol (Table 1).

Table 2 shows participants adherence to COVID-19 appropriate behavior other than healthcare setting. Of the 215 respondents, $54.8 \%$ always disinfect home, $78.6 \%$ wash hands frequently with soap or hand sanitizer, and $82.3 \%$ cover mouth and nose when coughing or sneezing. Only 
TABLE 4 | Logistic regression analyses for association between appropriate behaviors and socio-demographic characteristics of the study participants ( $N=215)$.

"Adherence to COVID-19 appropriate behaviors"

\begin{tabular}{lllll}
\hline \multicolumn{2}{c}{ At workplace } & & \multicolumn{2}{c}{ At home } \\
& aOR $[95 \% \mathrm{Cl}]$ & & OR $[95 \% \mathrm{Cl}]$ & aOR $[95 \% \mathrm{Cl}]$
\end{tabular}

\section{Risk perceptions}

Low

Moderate

High

Age

18-25

Above 26

Gender

Male

Female

Marital status

Single

Married

Nationality

Saudi

Non-Saudi

City of residence

Central

East

West

South

Highest education

Bachelors

Masters and above

Workplace

Government

Private

Work history

$<5$ years

5 years and above

Direct care

No

Yes

${ }^{\star} P<0.01$
Ref

1.38 [0.82-2.32]

$2.56[1.08-3.48]$ *

Ref

1.73 [0.91-2.96]

Ref

1.13 [0.85-2.75]

Ref

$1.36[1.08-2.41]$ *

Ref

1.27 [0.89-1.80]

Ref

1.14 [0.66-1.33]

1.21 [0.79-2.55]

1.35 [0.96-2.40]

Ref

1.65 [0.96-2.42]

Ref

$1.18[0.74-2.46]$

Ref

2.00 [1.13-3.80] *

Ref

1.21 [0.89-2.93]
1.20 [0.93-2.90]

1.35 [0.97-2.90]

$2.32[1.09-3.27]$ *

1.85 [0.98-3.63]

1.35 [1.07-2.30] *

1.35 [0.96-1.90]

1.15 [0.99-2.00]

1.25 [0.78-2.33]

1.32 [0.79-2.46]

1.68 [0.94-2.51]

1.32 [0.90-2.47]

2.08 [1.14-3.15] *

1.30 [0.91-2.83]
Ref

1.25 [0.93-2.56]

$2.65[1.15-4.61]^{*}$

1.22 [0.98-2.31]

$2.81[1.13-4.50]^{*}$

Ref

1.72 [0.91-3.10]

1.60 [0.97-3.41]

Ref

1.27 [0.95--2.84]

1.30 [0.98-2.68]

Ref

$1.70[1.03-3.17]$ *

$1.68[1.06-2.95]$ *

Ref

1.17 [0.79-1.74]

1.35 [0.96-1.90]

Ref

1.20 [0.80-2.13]

1.22 [0.99-2.15]

1.23 [0.79-1.92]

1.25 [0.66-2.43]

1.41 [0.90-2.00]

1.45 [0.91-2.20]

Ref

1.50 [0.86-2.78]

1.58 [0.93-2.75]

Ref

1.19 [0.70-2.60]

$1.21[0.78-2.85]$

Ref

$1.96[1.21-3.70]$ *

2.12 [1.10-3.20] *

Ref

1.41 [0.85-2.80]

1.40 [0.90-2.92]
$47.4 \%$ reported that they wear mask at home. Most of them $(80.0 \%)$ avoided public transportation, and $69.3 \%$ social activities. About half of the study participants reported that they prefer not to visit hospital/clinic during the pandemic. Approximately $72.5 \%$ reported that they will prefer to avoid contact with people who recently had a travel history, and $66.5 \%$ avoided going to crowded places. Approximately $81.4 \%$ avoided contact with people who had fever or symptoms of respiratory illness, and $83.7 \%$ avoided shaking hands (Table 2).

Table 4 depicts the logistic regression analyses for association between protective behaviors and socio-demographic characteristics of the study participants. The multivariable logistic regression model indicated that risk perception of participants to COVID-19 infection was significantly predicting the protective behavior at workplace $(\mathrm{aOR}=$
2.32, $p<0.01)$, and non-workplace settings $(\mathrm{aOR}=2.81$, $p<0.01)$. Participants who were married had 1.68 times more likely to practice protective behavior at home $(\mathrm{aOR}=$ 1.68, $p<0.01)$. Similarly, participants who had a work history of 5 years and more were found to be adherence to the recommended protective behavior in both the settings (Table 4).

\section{DISCUSSIONS}

To the best of our knowledge this is the first study that provides evidence of adherence to precautionary behavioral practice toward COVID-19 among RTs in the Kingdom of Saudi Arabia and elsewhere. These healthcare professionals are found to be extremely valuable during the COVID-19 pandemic, and 
understanding their behavioral practices is extremely critical for policy implications. The findings of this study are vital for risk management and healthcare policy in the Kingdom and elsewhere. The study reported that most (80.9\%) of the RC professionals followed recommended practice at workplace to the highest level. However, the appropriate behavioral practice toward COVID-19 at home or non-healthcare settings was found to be compromised (65.0\%). The multivariable logistic regression analysis indicated a positive association between the higher risk perception toward the COVID-19 infection with adherence to precautionary behavior practices at both settings.

Since this is the first study in its nature, a direct comparison is limited. A qualitative comparison can be made with other groups of healthcare professionals. The risk perception and adherence level to the recommended preventive strategies among the study participants in this study was found to be much higher than the study reported elsewhere (17-20). A study conducted in China indicated that $89 \%$ of the healthcare providers are knowledgeable regarding preventive methods of COVID-19 (21). A study conducted in Ethiopia highlighted that there was a gap on recommended precautionary measures, especially for wearing masks and gloves among the healthcare professionals (17). The study reported that well-educated participants better exercise protective behavior (17).

Adherence to preventive measures for COVID-19 in Saudi Arabia among general population was found to be moderately high (22-24). A cross-sectional study conducted during the same periods to our study reported that participants aged 25-34 years were $25 \%$ less likely to comply with hand hygiene and social distancing (24).

The lower levels of adherence of COVID-19 appropriate behavior among the study participants in home environment needs further investigation, may be attributable to the poor habit of PPE wearing, a perception that they interfere with performing tasks. The scientific understanding of precautionary behavioral practice of among RTs is beyond the scope of the current study.

The key strengths of this study are that we used an appropriate random sample of participants across the country; the outcome variables were measured using a Likert scale than dichotomized response (Yes/No); and the study reported the behavioral practices at both settings-home, and workplace of the study participants. Some limitations to consider in the study are we relied on self-reported data of the participants who might be influenced by their RC specialty to self-report applications of high-protective measures. We have not accounted some vital information such as history of infection and vaccination among them or their families or coworkers, which potentially affect the behaviors of the participants. Next, discussing theories that could explain the behaviors beyond scope of the paper. The reported behavior could vary overtime, since the data were collected during the first wave of the pandemic, it will be interesting to study the change of behavior with the progress of the pandemic. In spite of the aforementioned limitations, the study has several advantages and warrants further investigation of this unique healthcare professional.

\section{CONCLUSION}

The study findings present a concerning outlook for the RT, a group continually needed at the forefront against COVID-19. The adherence level to recommended precautionary behavioral practice toward COVID-19 among the participants on the study was found to be much higher. Although most the participants reported recommended protective measures to the highest level at workplace, there was a gap on adherence to the appropriate measures at non-workplace, especially, behavioral practice for wearing masks and disinfecting homes. Furthermore, the finding also indicated a statistically significant positive association between the risk perception and adherence of the participants to recommended behavioral practices. Therefore, there is a need to educate the importance of precautionary measures in the non-healthcare settings.

\section{DATA AVAILABILITY STATEMENT}

The raw data supporting the conclusions of this article will be made available by the authors, without undue reservation.

\section{ETHICS STATEMENT}

Ethical approval was obtained from The Deanship of Scientific Research (DSR) at Imam Abdulrahman Bin Faisal University (IAU), Dammam, Saudi Arabia. All participants provided their written informed consent to participate in this study.

\section{AUTHOR CONTRIBUTIONS}

NA and BP conceptualized the study. NA conducted research, provided research materials, collected and organized data, as well as contributed substantially to writing the manuscript. BP analyzed and interpreted data and wrote the first draft of the article. All the authors have critically reviewed and approved the final draft and are responsible for the content and similarity index of the manuscript.

\section{FUNDING}

This research is funded by The Deanship of Scientific Research at Imam Abdulrahman Bin Faisal University (grant number Covid19-2020-031-CAMS).

\section{ACKNOWLEDGMENTS}

The authors want to express their profound thanks to the Imam Abdulrahman Bin Faisal University (IAU), Dammam, Saudi Arabia for providing the research grant for this study. We also acknowledge the study participants and numerous volunteers for their generous participation in the study. 


\section{REFERENCES}

1. Hester TB, Cartwright JD, DiGiovine DG, Karlic KJ, Kercheval JB, DiGiovine B, et al. Training and deployment of medical students as respiratory therapist extenders during COVID-19. ATS Sch. (2020) 1:14551. doi: 10.34197/ats-scholar.2020-0049PS

2. Roberts KJ, Johnson B, Morgan HM, Vrontisis JM. Evaluation of respiratory therapist extender comfort with mechanical ventilation during COVID-19 pandemic. Respir. (2021) 66:199-204. doi: 10.4187/respcare.08 459

3. Al-Otaibi $\mathrm{H}$, AlAhmari $\mathrm{M}$. The respiratory care profession in Saudi Arabia: Past and present. Ann Thorac Med. (2016) 11:237. doi: 10.4103/1817-1737.191872

4. Alotaibi G. Status of respiratory care profession in Saudi Arabia: A national survey. Ann Thorac Med. (2015) 10:55-60.

5. Nguyen LH, Drew DA, Graham MS, Joshi AD, Guo C-G, Ma W, et al. Risk of COVID-19 among front-line health-care workers and the general community: a prospective cohort study. Lancet Public Heal. (2020) 5:e47583. doi: 10.1016/S2468-2667(20)30164-X

6. Giannis D, Geropoulos G, Matenoglou E, Moris D. Impact of coronavirus disease 2019 on healthcare workers: beyond the risk of exposure. Postgrad Med J [Internet]. (2021) 97:326-8. doi: 10.1136/postgradmedj-2020-137988

7. Wu A, Huang X, Li C, Li L. Novel coronavirus (2019-nCov) pneumonia in medical institutions: problems in prevention and control. Chin J Infect Control. (2020) 19:99-104. doi: 10.1148/radiol.2020200274

8. Li Y, Scherer N, Felix L, Kuper H. Prevalence of depression, anxiety and post-traumatic stress disorder in health care workers during the COVID-19 pandemic: A systematic review and meta-analysis. Pietschnig J, editor PLoS One. (2021) 16:e0246454. doi: 10.1371/journal.pone.0246454

9. Mirtskhulava V, Whitaker JA, Kipiani M, Harris DA, Tabagari N, Owen-Smith $\mathrm{AA}$, et al. Determinants of tuberculosis infection control-related behaviors among healthcare workers in the country of Georgia. Infect Control Hosp Epidemiol [Internet]. (2015) 36:522-8. doi: 10.1017/ice.2015.5

10. Tenna A, Stenehjem EA, Margoles L, Kacha E, Blumberg HM, Kempker RR. Infection control knowledge, attitudes, and practices among healthcare workers in Addis Ababa, Ethiopia. Infect Control Hosp Epidemiol. (2013) 34:1289-96. doi: 10.1086/673979

11. Saunders-Hastings P, Crispo JAG, Sikora L, Krewski D. Effectiveness of personal protective measures in reducing pandemic influenza transmission: A systematic review and meta-analysis. Epidemics. (2017) 20:1-20 doi: 10.1016/j.epidem.2017.04.003

12. World Health Organization. Health Workers Exposure Risk Assessment and Management in the Context of COVID-19 Virus: Interim Guidance. (2020). Avaliable online at: https://apps.who.int/iris/bitstream/handle/10665/331340/ WHO-2019-nCov-HCW_risk assessment-2020.1-eng.pdf

13. Conner M, Norman P. Predicting health behavior. London, UK: McGraw-Hill Education (UK). (2005).

14. Diefenbach MA, Leventhal $H$. The common-sense model of illness representation: Theoretical and practical considerations. J Soc distress homeless. (1996) 5:11-38. doi: 10.1007/BF02090456

15. Broadbent E, Petrie KJ, Main J, Weinman J. The brief illness perception questionnaire. J Psychosom Res. (2006) 60:6317. doi: 10.1016/j.jpsychores.2005.10.020

16. MILNE S, SHEERAN P, ORBELL S. Prediction and intervention in health-related behavior: a meta-analytic review of protection motivation theory. J Appl Soc Psychol [Internet]. (2000) 30:10643. doi: 10.1111/j.1559-1816.2000.tb02308.x

17. Girma S, Alenko A, Agenagnew L. Knowledge and precautionary behavioral practice toward COVID-19 among health professionals working in public university hospitals in Ethiopia: a web-based survey. Risk Manag Healthc Policy [Internet]. (2020) 13:1327-34. doi: 10.2147/RMHP.S267261

18. Kamel G, Sakineh, Rakhshanderou Fatemeh H, Mohtasham G. COVID-19 protective behaviors among iran's health system workers : psychological theoretic design analysis. BMC Health Serv Res. (2021) 1-13. doi: 10.21203/rs.3.rs-151581/v1

19. Tabah A, Ramanan M, Laupland KB, Buetti N, Cortegiani A, Mellinghoff J, et al. Personal protective equipment and intensive care unit healthcare worker safety in the COVID-19 era (PPE-SAFE): An international survey. J Crit Care [Internet]. (2020) 59:70-5. doi: 10.1016/j.jcrc.2020.06.005

20. Wong EL-Y, Ho K-F, Dong D, Cheung AW-L, Yau PS-Y, Chan EY-Y, et al. Compliance with standard precautions and its relationship with views on infection control and prevention policy among healthcare workers during COVID-19 pandemic. Int J Environ Res Public Health [Internet]. (2021) 18:3420. doi: 10.3390/ijerph18073420

21. Zhang M, Zhou M, Tang F, Wang Y, Nie H, Zhang L, et al. Knowledge, attitude, and practice regarding COVID-19 among healthcare workers in Henan, China. J Hosp Infect [Internet]. (2020) 105:183-7. doi: 10.1016/j.jhin.2020.04.012

22. Alghamdi AN, Alotaibi MI, Alqahtani ASA, Al Aboud D, Abdel-Moneim AS. Association between educational status and awareness of adherence to preventive measures for COVID-19 in Saudi Arabia. Asia Pacific J Public Heal [Internet]. (2021) 6:101053952110146. doi: 10.1177/1010539521101 4637

23. Alyami H, Alyami $M$, Krägeloh C, Nemenqani D, Henning $M$. Coping strategies during the COVID-19 pandemic and compliance with precautionary health behaviors: An online study among the Saudi adult population. Saudi J Heal Sci [Internet]. (2021) 10:21. doi: 10.4103/sjhs.sjhs_222_20

24. AL-Shammary AA, Hassan S un-N, Zahra A, Algahtani FBZ, Suleiman S. Role of community-based measures in adherence to self-protective behaviors during first wave of COVID-19 pandemic in Saudi Arabia. Heal Promot Perspect [Internet]. (2021) 11:69-79. doi: 10.34172/hpp.2021.10

Conflict of Interest: The authors declare that the research was conducted in the absence of any commercial or financial relationships that could be construed as a potential conflict of interest.

Publisher's Note: All claims expressed in this article are solely those of the authors and do not necessarily represent those of their affiliated organizations, or those of the publisher, the editors and the reviewers. Any product that may be evaluated in this article, or claim that may be made by its manufacturer, is not guaranteed or endorsed by the publisher.

Copyright (C) 2021 Al Khathlan and Padhi. This is an open-access article distributed under the terms of the Creative Commons Attribution License (CC BY). The use, distribution or reproduction in other forums is permitted, provided the original author(s) and the copyright owner(s) are credited and that the original publication in this journal is cited, in accordance with accepted academic practice. No use, distribution or reproduction is permitted which does not comply with these terms. 